\title{
A Segmented Anode Vacuum Phototriode with Position Sensitivity
}

\author{
S. Zahid, ${ }^{\mathrm{a}, 1}$ P. R. Hobson, ${ }^{\mathrm{a}}$ D. J. A. Cockerill ${ }^{\mathrm{b}}$
}

${ }^{a}$ Brunel University London,

Kingston Lane, London, Uxbridge UB8 3PH, UK

${ }^{\mathrm{b}}$ Rutherford Appleton Laboratory,

Harwell, Oxford, Didcot OX11 OQX, UK

E-mail: Sema.Zahid@brunel.ac.uk

\begin{abstract}
A new four-fold segmented multi-anode vacuum phototriode is presented. A simulation using the COMSOL multi-physics simulation software is described and the predicted induced signals on the anode and the inter-anode cross-talk calculated. Experimental data on the spatial uniformity of the response across the photocathode, the cross talk between segments and the dark currents at room temperature are presented. The response of the VPT at 14 degrees to a magnetic field of up to $4 \mathrm{~T}$ is determined.
\end{abstract}

Keywords: Electron multipliers (vacuum), Photon detectors for UV, visible and IR photons (vacuum)

\section{Contents}

1 Introduction 1

2 Segmented Anode VPT 2

2.1 Construction 2

${ }^{1}$ Corresponding author. 
3 Simulation 2

3.1 VPT simulation 2

3.2 Simulation Results 3

4 Experimental Results 4

4.1 Scan of VPT photocathode response 4

4.2 VPT operation in a Magnetic Field 4

4.3 Results 5

4.3.1 Anode Response Uniformity and Crosstalk

$\begin{array}{ll}\text { 4.3.2 Response in high Magnetic Fields } & 7\end{array}$

5 Conclusion $\quad 8$

\section{Introduction}

Vacuum phototriodes (VPTs) have been used for many years in particle physics experiments. For example, they were used in the OPAL experiment [1] at LEP and are currently used in the endcap electromagnetic calorimeter of the CMS experiment [2] at CERN's Large Hadron Collider.

VPTs are fast, proximity focused devices. They comprise an anode or dynode that can be subdivided into several independent channels within the same overall vacuum envelope. Such a device could be useful for reading out multiple scintillating or wavelength shifting fibres from a "SPACAL" type of calorimeter [3].

Hamamatsu have constructed a prototype segmented VPT for us (type "Triode4", serial number - ZG6968) that is configured with a fine mesh, transmissive dynode followed by a fourfold segmented anode. The tube has a standard bialkali photocathode with a quoted response of "about $60 \mu \mathrm{A} / \mathrm{lm}$ ". This is lower than a typical mass production PMT with this photocathode. The novelty of this VPT is that it contains four independent channels sharing a common photocathode and gain stage. This is beneficial for applications with limited space, as it minimises the inactive area compared to four smaller independent devices; significant cost savings are also likely to accrue.

This paper presents an experimental characterisation of the new segmented anode VPT and gives predictions for the pulse shape and cross-talk using a COMSOL Multiphysics simulation. An area scan at $0.00 \mathrm{~T}$ across the entire photocathode, and a fine line scan to determine the intersegment cross talk, is presented. Measurements of the relative response of the VPT in quasi-axial magnetic fields up to $4 \mathrm{~T}$, with respect to $0.00 \mathrm{~T}$, are described.

\section{Segmented Anode VPT}

\subsection{Construction}

The prototype VPT is $40 \mathrm{~mm}$ long, with a $23 \mathrm{~mm}$ external diameter vacuum envelope. The anode is split into four equal quadrants with a nominal $1 \mathrm{~mm}$ gap between the straight edges of each 
quadrant. The four segments are labelled A1 to A4, as shown in Figure 1. The separations between the cathode and dynode, and the dynode to anode are not provided by Hamamatsu. They are difficult to determine accurately without destroying the VPT. Separations of $4.0 \mathrm{~mm}$ and $3.0 \mathrm{~mm}$ respectively have been used in the COMSOL model. These values are accurate to $0.5 \mathrm{~mm}$.
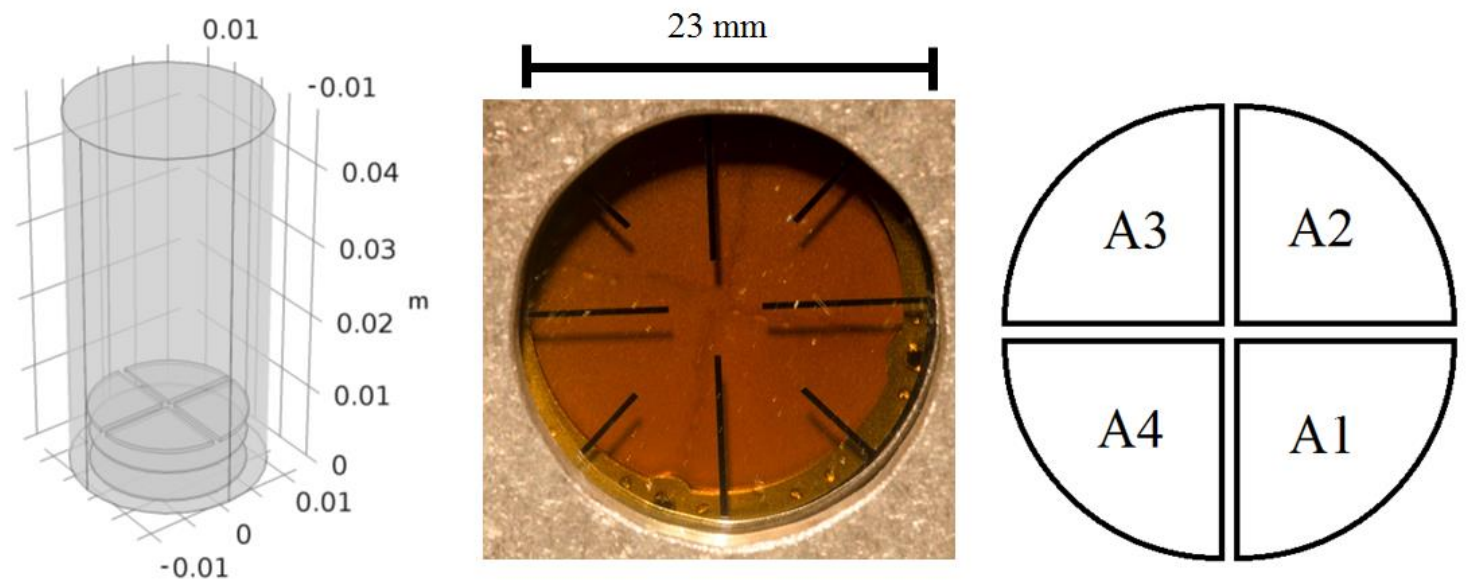

Figure 1. The segmented anode VPT. Left, the implementation of the device in the COMSOL simulation to scale (40 $\mathrm{mm}$ long and $23 \mathrm{~mm}$ in diameter). Centre, a photograph of the VPT showing the conductive lines on the photocathode, with the dynode behind. Right, the anode segment numbering used in this paper.

\section{Simulation}

This section covers the modelling and simulation of the segmented VPT using the COMSOL Multiphysics software [4].

\subsection{VPT simulation}

The VPT model is implemented within COMSOL version 5.3. A three-dimensional geometry at $1: 1$ scale, for each component within the VPT, is created. Materials and properties are assigned to the components, together with their operational properties such as their electric potential. Finally, a tetrahedral mesh is applied to the whole model.

The prototype VPT contains a transmissive dynode constructed using a very fine metal mesh. For the simulation this is created as a thin, solid disc that operates as a transmissive electrode. The cathode is set to $0 \mathrm{~V}$, the dynode to $600 \mathrm{~V}$ and the anode to $800 \mathrm{~V}$. The materials for the components are matched to the prototype based on the closest available selections in the COMSOL library. The dynode and cathode (materials) are set to aluminium gallium arsenide. The anode is set to beryllium copper. The vacuum space is created by modifying the properties of air. The simulation evaluates the electric field distribution inside the VPT, shown in Figure 2. Electrons are then released from the photocathode and the induced currents on the anodes calculated by applying Ramo's theorem at each time step [5]. 


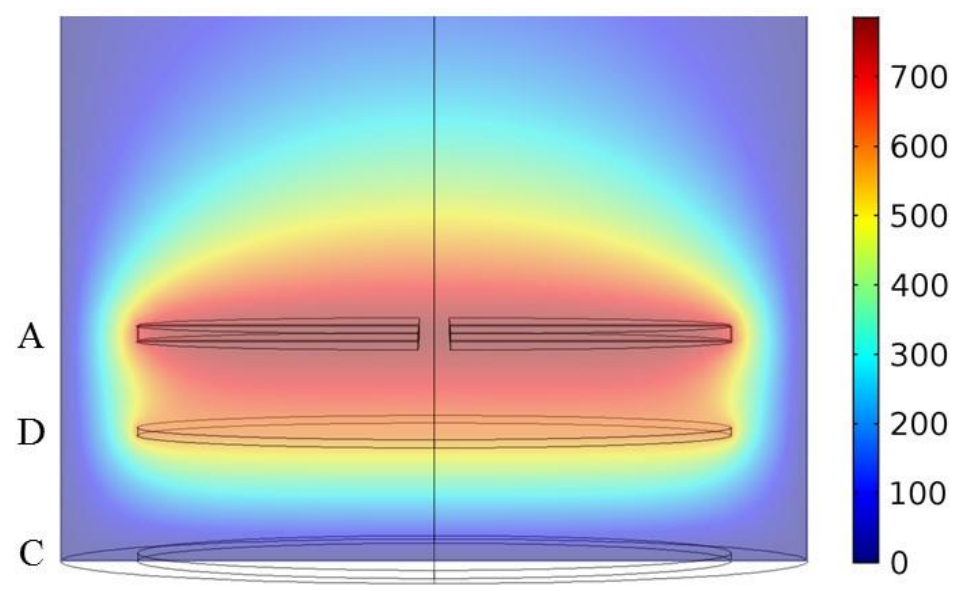

Figure 2. Longitudinal cross section of the VPT showing the electric potential. The coloured scale shows the electric potential in volts.

\subsection{Simulation Results}

Figure 3 (left) shows the induced current from a direct hit on an anode segment, for an electron emitted from the photocathode with the VPT in a $0.00 \mathrm{~T}$ magnetic field, and a secondary electron with the VPT in a uniform $4.00 \mathrm{~T}$ magnetic at $15^{\circ}$ to the VPT axis of cylindrical symmetry. This configuration was chosen to compare with existing VPTs used in the CMS endcap calorimeters. The peak induced current is $\sim 0.5 \mathrm{nA}$, with the magnetic field having a minimal effect. Figure 3 (right) shows the induced current crosstalk on an adjacent segment. The magnetic field causes a distinct difference in the predicted cross-talk signal. The point of particle release from the photocathode is the same for both sets of simulations. However, for the cross-talk simulation $4 \mathrm{~T}$ with a $15^{\circ}$ field, due to its magnetic field influence on the initial photoelectron, the secondary electron is released from a different point from the dynode when compared to $0.00 \mathrm{~T}$. It is important to note that our model has certain approximations. The dynode is assumed to be uniform whereas, in reality, it is a very fine metal mesh of unknown construction. Multi-dynode tubes using fine transmissive meshes do show a significant decrease in gain in strong magnetic fields [6].

The simulation predicts that, as expected, the VPT is an intrinsically fast photodetector, with rise and fall times of $1 \mathrm{~ns}$ or less. 

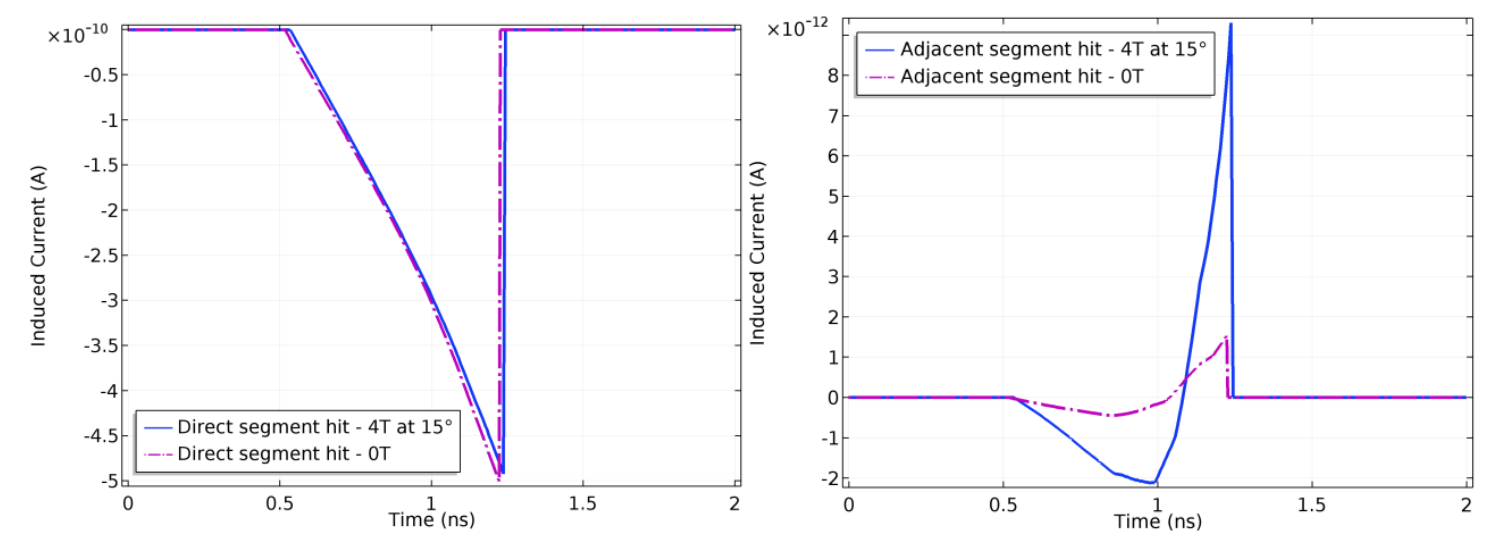

Figure 3. Simulation of the induced current of a single secondary electron for a VPT at $0 \mathrm{~T}$ and at $15^{\circ}$ to a 4 $\mathrm{T}$ field. Left, the current arising from a direct hit on an anode segment. Right, the cross-talk induced current arising from a hit on an adjacent segment.

\section{Experimental Results}

\subsection{Scan of VPT photocathode response}

The DC response of the prototype was measured at $0.00 \mathrm{~T}$ by scanning across the entire photocathode for each anode segment. The VPT was mounted on an X-Y-Z stage located in a dark optics lab. A motorised $\mathrm{x}$-axis stage was controlled through a Newport ESP3000 Motion Controller. The y-axis was controlled manually with a micrometre. The cathode was set to -800 $\mathrm{V}$, the dynode to $-200 \mathrm{~V}$ and the four anodes to $0 \mathrm{~V}$.

A blue LED (Kingbright L-2523QBC-D) was used as the light source. Our LED has a measured peak wavelength of $488 \mathrm{~nm}$. The light was focused onto a $500 \mu \mathrm{m}$ pinhole, with a second biconvex lens to refocus the light onto the photocathode. The FWHM of the focused spot was determined, using a CMOS pixel imaging device, to be $370 \mu \mathrm{m}$. The power at the focus was 106 $\mathrm{nW}$.

Figure 4 shows a full scan was made across the VPT faceplate using $1.00 \mathrm{~mm}$ steps in both $\mathrm{x}$ and y directions. A separate, fine resolution, scan was also carried out across an $8.5 \mathrm{~mm} \times 3 \mathrm{~mm}$ region with a step size of $0.25 \mathrm{~mm}$. This is smaller than the FWHM of the light spot, in order to obtain a detailed measurement of the transition that occurs between adjacent anodes. During this scan, the induced signal (crosstalk) was also measured across the three adjacent anodes.

\subsection{VPT operation in a Magnetic Field}

The magnetic field tests were carried out in a warm bore $4 \mathrm{~T}$ superconducting solenoidal magnet. The prototype VPT was placed within an electrically shielded cylinder with two Nichia NSPB 500S LEDs to illuminate the photocathode approximately uniformly. The LEDs were driven with a square voltage wave at $100 \mathrm{kHz}$. The LEDs emit blue light with a peak wavelength of $470 \mathrm{~nm}$ and FWHM of $30 \mathrm{~nm}$. The VPT response was measured at $4.00 \mathrm{~T}, 1 \mathrm{~T}$ and $0.00 \mathrm{~T}$, with the angle of the axis of the VPT set to either $14 \pm 1^{\circ}$ or $0 \pm 1^{\circ}$. The VPT was placed in the solenoidal fringe field for the $1 \mathrm{~T}$ measurements, which varies from $0.97 \mathrm{~T}$ to $1.25 \mathrm{~T}$ over a distance of $10 \mathrm{~mm}$ along 
the VPT longitudinal axis. The $0.00 \mathrm{~T}$ measurements were carried out with the same equipment, in the region where the field was measured to be $<0.1 \mathrm{mT}$.

\subsection{Results}

\subsubsection{Anode Response Uniformity and Crosstalk at 0.00 T}

The results from the faceplate scan are shown as a heat map in Figure 4. The response of each anode segment has been normalised to its own peak current. The heat map shows a higher response towards the centre of each segment, with peak currents of about $105 \mathrm{nA}$. The internal structure of the VPT is visible in the scan. The physical gaps between each anode segment give rise to lower response. Diagonal regions of lower response are seen in each anode segment, for example at locations $(5,18),(6,17),(7,16)$ in anode segment $A 4$. These are caused by the thin metal electrodes on the faceplate shown in Figure 1.

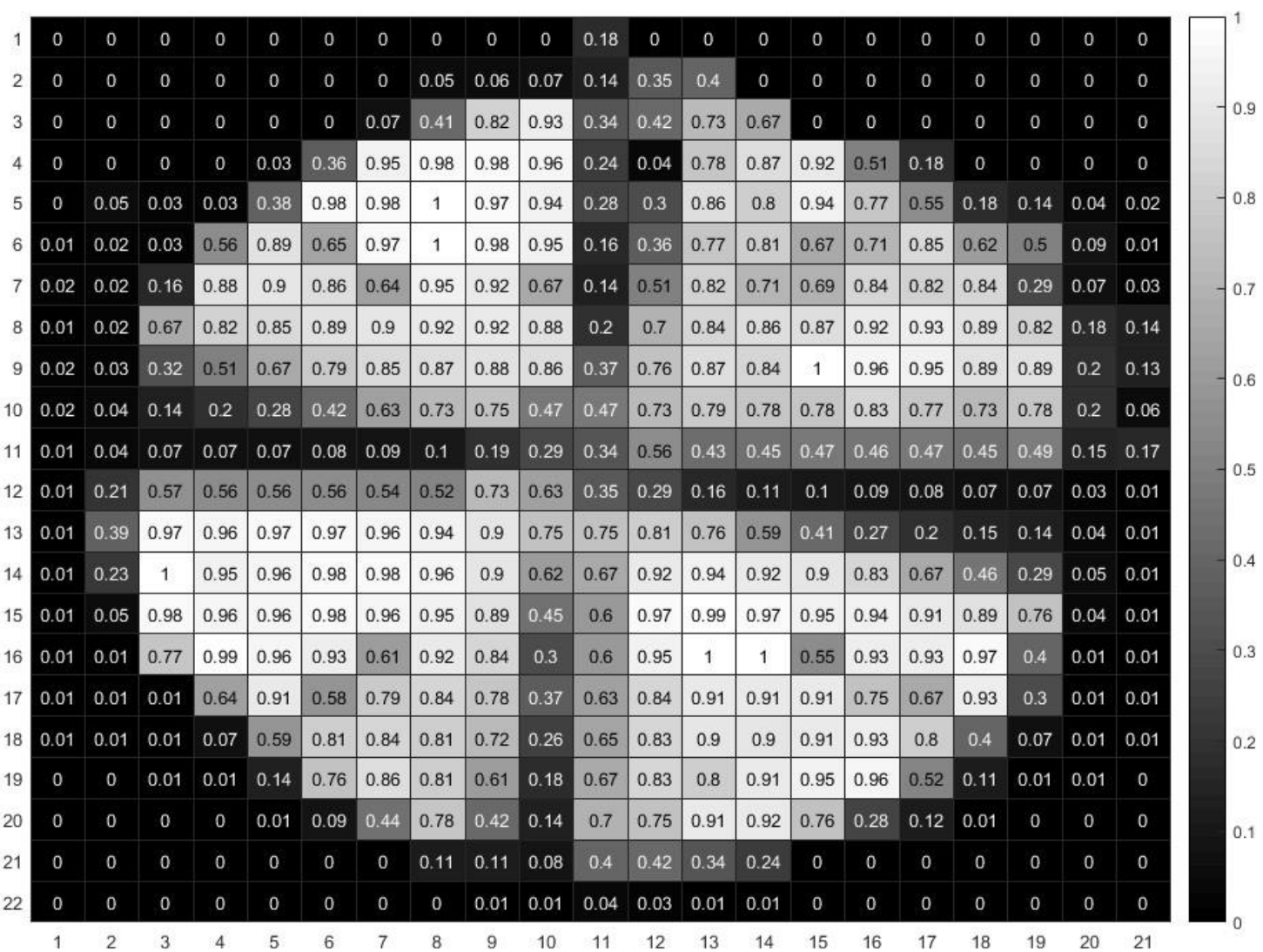

Figure 4. Heatmap normalised to the peak current in each anode separately. The pixel size is $1.00 \mathrm{~mm} \times$ $1.00 \mathrm{~mm}$

The response of anode segment A2 (upper-right in Figure 4) appears to be less uniform compared to the other segments. The peak response of anode A2 is nearly $30 \%$ higher than the other 3 anodes, for reasons unknown, although it is not due to excessive dark current. The peak currents are $\sim 105,133,103$, and $103 \mathrm{nA}$ for A1 to A4 respectively. The dark currents are $\sim 0.19$, $3.7,1.9$ and $0.6 \mathrm{nA}$ for $\mathrm{A} 1$ to A4 respectively. 
Crosstalk is a form of correlated noise across closely spaced components. Crosstalk could be an important consideration since the VPT has four anode segments separated by small gaps. Figure 5 (a) shows the crosstalk in segment A4 for LED light illuminating the photocathode above segment A1, and is quoted as the percentage of the current from anode A1. The crosstalk is $2-3 \%$ for electrons hitting the centre of A1. Figure 5 (b) shows the response across A1, which has a peak current of $105.5 \mathrm{nA}$. The data were taken in $1 \mathrm{~mm}$ steps in the $\mathrm{x}$ and $\mathrm{y}$ axes.

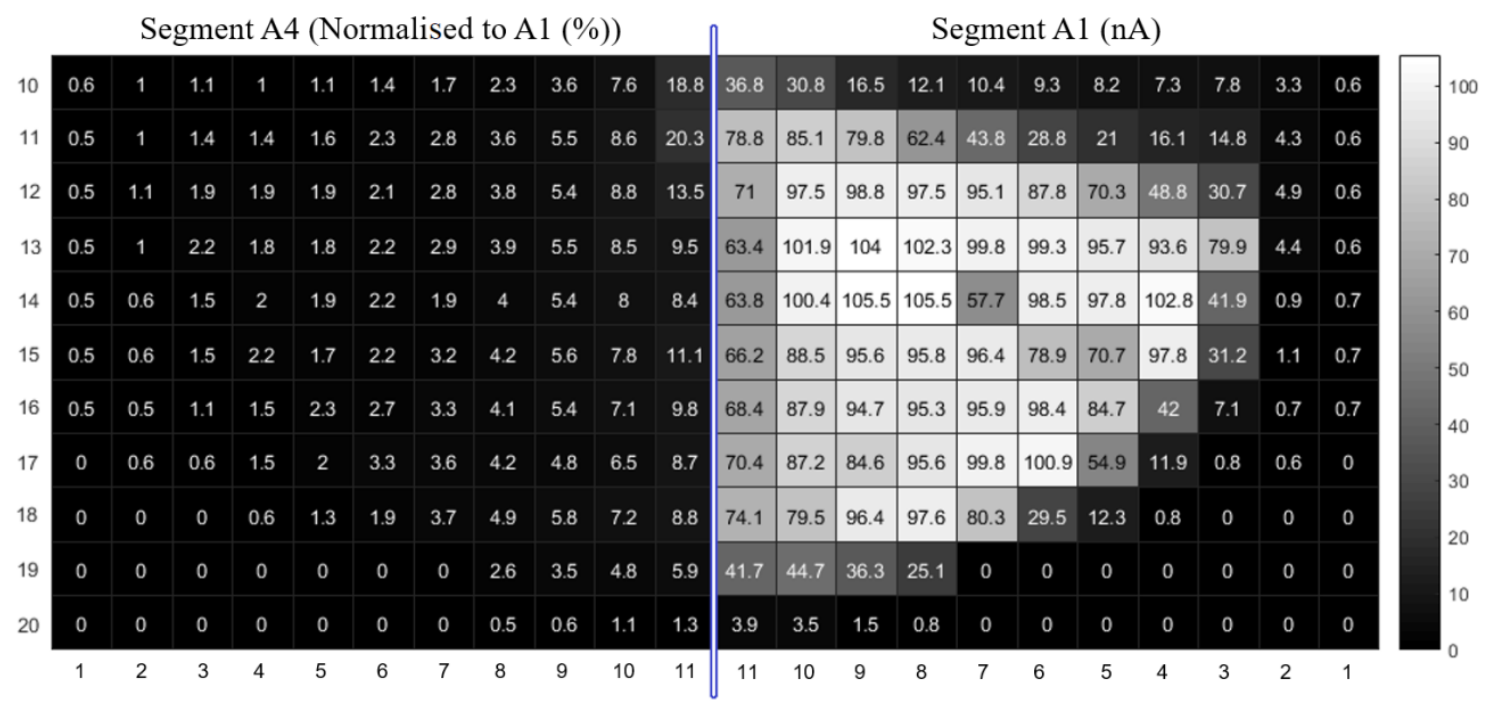

(a)

(b)

Figure 5. Heatmap showing the percentage crosstalk measured on anode A4 when the LED is focused on the photocathode in the region above anode A1. Heatmap (b) shows the output current of anode A1 in nA. The pixel size is $1.00 \mathrm{~mm} \times 1.00 \mathrm{~mm}$

Figure 6 shows three fine resolution line scans in the region highlighted across A4. The data indicate a generally uniform photocathode response. 
120

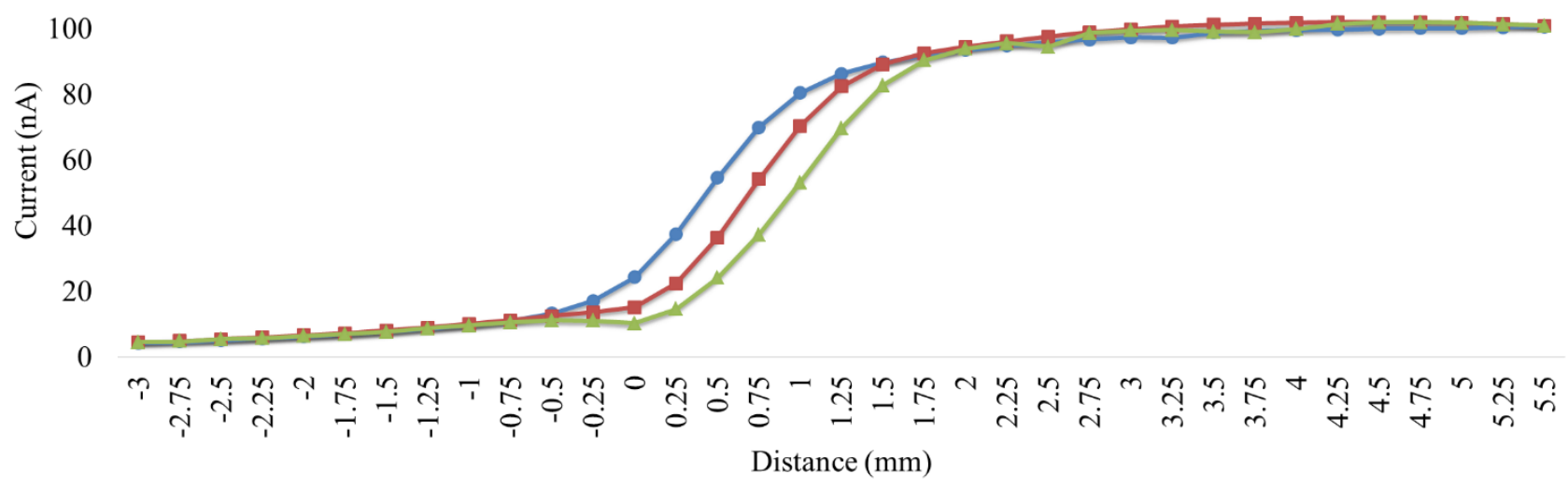

Figure 6. Fine scan in steps of $0.25 \mathrm{~mm}$ steps in the horizontal axis. The curve legends correspond to the rows of the heatmap in Figure 4.

\subsubsection{Response in high Magnetic Fields}

Figure 7 shows the response of each anode segment, normalised to its response at a dynode voltage of $50 \mathrm{~V}$, in magnetic fields of $0.00 \mathrm{~T}, 1 \mathrm{~T}$ and $4.00 \mathrm{~T}$, for orientations of $0^{\circ}$ and $14^{\circ}$ to the magnetic field. The photocathode was held at $0 \mathrm{~V}$ and all four anodes at $800 \mathrm{~V}$. The relative responses of all four anode segments are essentially identical as a function of the dynode voltage.
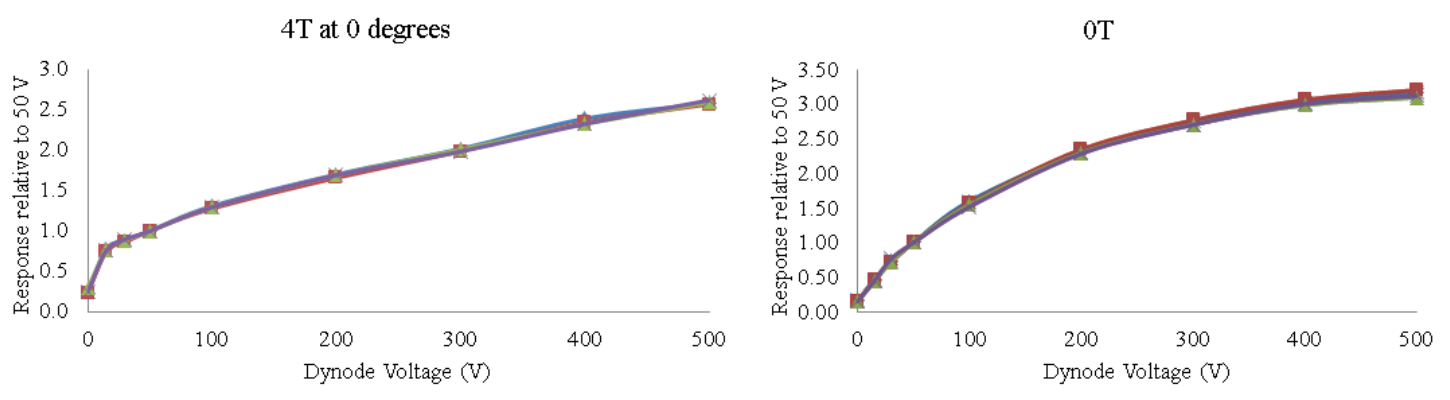

$1 \mathrm{~T}$ at 14 degrees

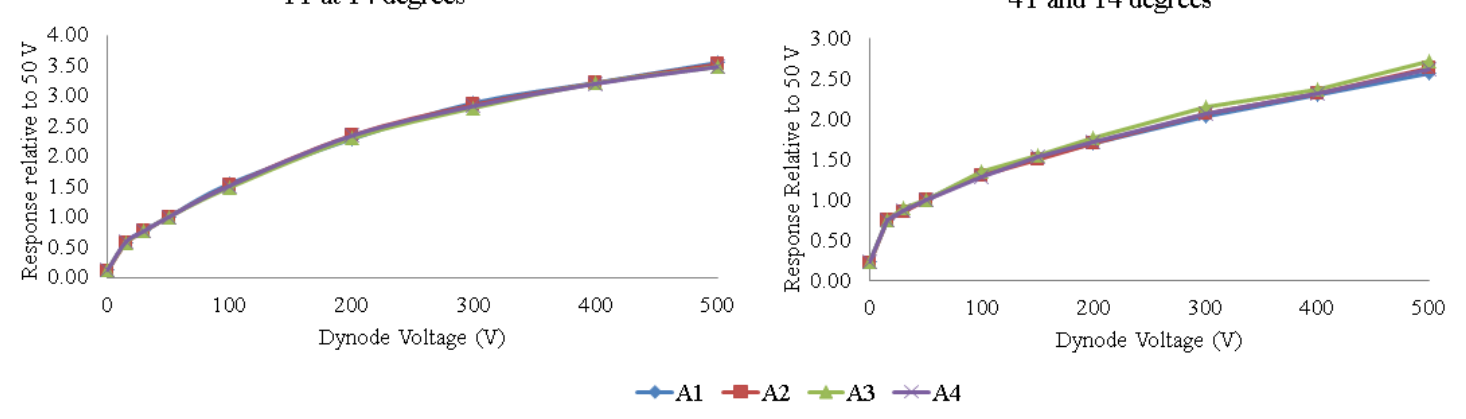

Figure 7. Anode response normalised to a dynode voltage of $50 \mathrm{~V}$ for the VPT in magnetic fields of 0.00 $\mathrm{T}, 4.00 \mathrm{~T}$ at $0^{\circ}$ to the VPT axis, and $1 \mathrm{~T}$ and $4.00 \mathrm{~T}$ at $14^{\circ}$ to the VPT axis. 
Figure 8 shows the ratio of the VPT response at $4.00 \mathrm{~T}$ to $0.00 \mathrm{~T}$ as a function of dynode voltage, at $0^{\circ}$ to the VPT axis. The response is a factor 6 lower in the magnetic field. The apparent improvement at very small cathode-to-dynode potential differences, is unexpected but may be due to the details of the fine dynode mesh. It is of limited practical advantage as the tube is operating at very low gain in this region.

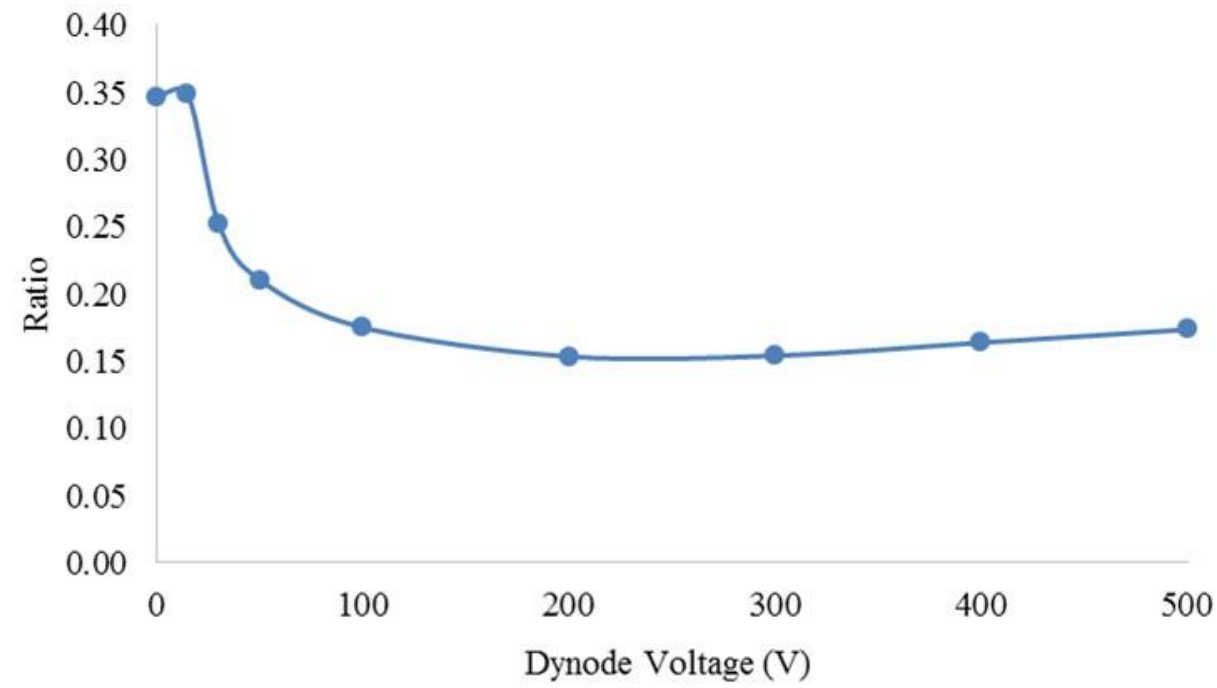

Figure 8. Ratio of the response at $4 \mathrm{~T}$ to $0.00 \mathrm{~T}$, as a function of dynode voltage, of all four anodes for the VPT at $0^{\circ}$ in a magnetic field of $4.00 \mathrm{~T}$ and $0.00 \mathrm{~T}$.

\section{Conclusion}

The characteristics of a Hamamatsu prototype segmented anode VPT have been measured. The response to a light spot scanned across the photocathode demonstrates that all four anodes operate independently, with minimal cross-talk until the light is within about $1 \mathrm{~mm}$ to the anode segment gaps. Measurements in a uniform, axial $4.00 \mathrm{~T}$ magnetic field show a significant drop in response by a factor of nearly six compared to the response at $0.00 \mathrm{~T}$.

The VPT response has been modelled using the simulation package COMSOL V 5.3. The time response and time-dependent cross-talk has been numerically calculated using an ideal dynode approximation. The simulation predicts that, as expected, the VPT should be an intrinsically fast photodetector with rise and fall times of the order of $1 \mathrm{~ns}$ or shorter.

Experimental measurements of the pulse response, using an $80 \mathrm{ps}$ laser diode light source, are planned.

\section{Acknowledgments}

One of us (S. Zahid) would like to acknowledge funding for this project via an STFC Doctoral Training award - ST/M503393/1. 
Data created during this research are openly available from a data archive at http://dx.doi.org/10.17633/rd.brunel.5620561 .

\section{References}

[1] M. Akrawy et al., "Development studies for the OPAL end cap electromagnetic calorimeter using vacuum photo triode instrumented leadglass," Nuclear Instruments and Methods in Physics Research Section A: Accelerators, Spectrometers, Detectors and Associated Equipment, vol. 290, no. 1, pp. 76-94, 1990.

[2] CMS Collaboration, "Performance and operation of the CMS electromagnetic calorimeter," Journal of Instrumentation, vol. 5, no. 03, p. T03010, 2010.

[3] N. Akchurin et al, "The electromagnetic performance of the RD52 fiber calorimeter," Nuclear Instruments and Methods in Physics Research Section A: Accelerators, Spectrometers, Detectors and Associated Equipment, vol. 735, pp. 130-144, 2014.

[4] “COMSOL Multiphysics ${ }^{\odot}$ V. 5.3, www.comsol.com," COMSOL AB, Stockholm, Sweden.

[5] W. Shockley, "Currents to conductors induced by a moving point charge," Journal of Applied Physics, vol. 9, no. 10, pp. 635-636, 1938.

[6] F. Takasaki, K. Ogawa and K. Tobimatsu, "Performance of a photomultiplier tube with transmissive dynodes in a high magnetic field," Nuclear Instruments and Methods in Physics Research Section A: Accelerators, Spectrometers, Detectors and Associated Equipment, vol. 228, no. 2-3, pp. 369-373, 1985. 